\title{
A time series model of CDS sequences in complete genome
}

\author{
Zu-Guo $\mathrm{Yu}^{1,2}$ and Bin Wang ${ }^{2}$ \\ ${ }^{1}$ Department of Mathematics, Xiangtan University, Hunan 411105, China. * \\ ${ }^{2}$ Institute of Theoretical Physics, Academia Sinica, \\ P.O. Box 2735, Beijing 100080, China.
}

\begin{abstract}
A time series model of CDS sequences in complete genome is proposed. A map of DNA sequence to integer sequence is given. The correlation dimensions and Hurst exponents of CDS sequences in complete genome of bacteria are calculated. Using the average of correlation dimensions, some interesting results are obtained.
\end{abstract}

PACS numbers: $87.10+\mathrm{e}, 47.53+\mathrm{n}$

Key words: Correlation dimension, Hurst exponent, complete genome,

\section{Introduction}

In the past decade or so there has been a ground swell of interest in unraveling the mysteries of DNA. With improving of the technique of gene clone and sequences determined, the DNA sequence data base become huge rapidly. Doing DNA sequence analysis only use the experimental method does not fit this rapid. Hence it becomes very important to improve new theoretical methods. One approach that has, in just a few years, proven to be particularly fruitful in this regard is statistical analysis of DNA sequences ${ }^{[1-9]}$ using modern statistical measures, including the works on the correlation properties of coding and noncoding DNA sequences. The second approach is linguistic approach. DNA sequence can be seen as analogous at a number of levels to mechanisms of processing other kinds of languages, such as natural languages and computer languages 10 . Third, using

\footnotetext{
*This is the permanent corresponding address of the first author, e-mail: yuzg@hotmail.com
} 
nonlinear scales method, such as fractal dimension 11, 12, 13, complexity 14, 15. However, DNA sequences are more complicated than these types of analysis can describes. Therefore, it is crucial to develop new tools for analysis with a view toward uncovering mechanisms used to code other types of information.

Since the first complete genome of a free-living bacterium Mycoplasma genitalium was sequenced in 1995 16 , an ever-growing number of complete genomes has been deposited in public databases. The availability of complete genomes opens the possibility to ask some global questions on these sequences. Our group also discussed the avoided and under-represented strings in some bacterial complete genomes 13, 17, 18. In this paper, we propose a new model to DNA sequences, i.e. the time series model. First we want to compute the correlation dimension and Hurst exponents of each CDS sequence in the complete genome, then consider the distribution of these two quantities on complete genomes of Bacteria. It is a global problem. Last we want to discuss the classification problem of Bacteria using our results.

For the present purpose, a DNA sequence may be regard as a sequence over the alphabet $\{A, C, G, T\}$ representing the four bases from which DNA is assembled, namely adenine, cytosine, guanine, and thymine. For a DNA sequence, we define a map $f$ as following:

$$
\begin{aligned}
A & \longrightarrow-2 \\
f: \quad & C-1 \\
G & \longrightarrow 1 \\
T & \longrightarrow 2 .
\end{aligned}
$$

Then we obtain an data sequence $\left\{x_{k}: k=1,2, \cdots, N\right\}$, where $x_{k} \in\{-2,-1,1,2\}$. We formal view this sequence as a time series. According to the definition of $f$, the four bases $\{A, C, G, T\}$ are mapped to four distinct value. One can also use $\{-2,-1,1,2\}$ to replace $\{A, G, C, T\}$ or other orders of $A, G, C, T$. our main aim is distinguish $A$ and $G$ from purine, $C$ and $T$ from pyrimidine. We expect it to reveal more information than one dimensional DNA walk 4 .

\section{Correlation dimension and Hurst exponent}

The notion of correlation dimension, introduced by Grassberger and Procaccia [19, 20], suits well experimental situations, when only a single time series is available, it is now being used widely in many branches of physical science. Given a sequence of data from a computer or laboratory experiment

$$
x_{1}, x_{2}, x_{3}, \cdots, x_{N}
$$


where $N$ is a big enough number. These number are usually sampled at an equal time interval $\Delta \tau$. We embed the time series into $\mathbf{R}^{m}$, choose a time delay $\tau=p \Delta \tau$, then obtain

$$
\mathbf{y}_{i}=\left(x_{i}, x_{i+p}, x_{i+2 p}, \cdots, x_{i+(m-1) p}\right), \quad i=1,2, \cdots, N_{m}
$$

where

$$
N_{m}=N-(m-1) p .
$$

In this way we get $N_{m}$ vectors of embedding space $\mathbf{R}^{m}$.

For any $\mathbf{y}_{i}, \mathbf{y}_{j}$, we define the distance as

$$
r_{i j}=d\left(\mathbf{y}_{i}, \mathbf{y}_{j}\right)=\sum_{l=0}^{m-1}\left|x_{i+l p}-x_{j+l p}\right| .
$$

If the distance is less than a present number $r$, we say that these two vectors are correlated. The correlation integral is defined as

$$
C_{m}(r)=\frac{1}{N_{m}^{2}} \sum_{i, j=1}^{N_{m}} H\left(r-r_{i j}\right)
$$

where $H$ is the Heaviside function

$$
H(x)= \begin{cases}1, & \text { if } x>0 \\ 0, & \text { if } x \leq 0\end{cases}
$$

For a proper choice of $m$ and not too big a value of $r$, it has been shown by Grassberger and Procaccia 20 that the correlation integral $C_{m}(r)$ behaves like

$$
C_{m}(r) \propto r^{D_{2}(m)}
$$

Thus one can define correlation dimension as

$$
D_{2}=\lim _{m \longrightarrow \infty} D_{2}(m)=\lim _{m \rightarrow \infty} \lim _{r \longrightarrow 0} \frac{\ln C_{m}(r)}{\ln r} .
$$

For more details of $D_{2}$, the reader can refer to ref.[21].

To deal with practical problem, one usually choose $p=1$. From Page 346 of ref. 21], if we choose an sequence $\left\{r_{i}: 1 \leq i \leq n\right\}$ such that $r_{1}<r_{2}<r_{3}<\cdots<r_{n}$, then in the $\ln r-\ln C_{m}(r)$ plane, we can look for a scaling region. Then the slop of the scaling region is $D_{2}(m)$. When $D_{2}(m)$ dose not change with $m$ increasing, we can take this $D_{2}\left(m_{0}\right)$ as the estimate value of $D_{2}$. We calculate the correlation dimension of some DNA sequences using the method introduced above. From the $\ln r-\ln C_{m}(r)$ figures of these sequences of different value of embedding dimension $m$, we find that it is suitable to choose $m=7$. For example, we give the $\ln r-\ln C_{m}(r)$ figure of Phage's 5'UTR sequence when $m=7,8$ 


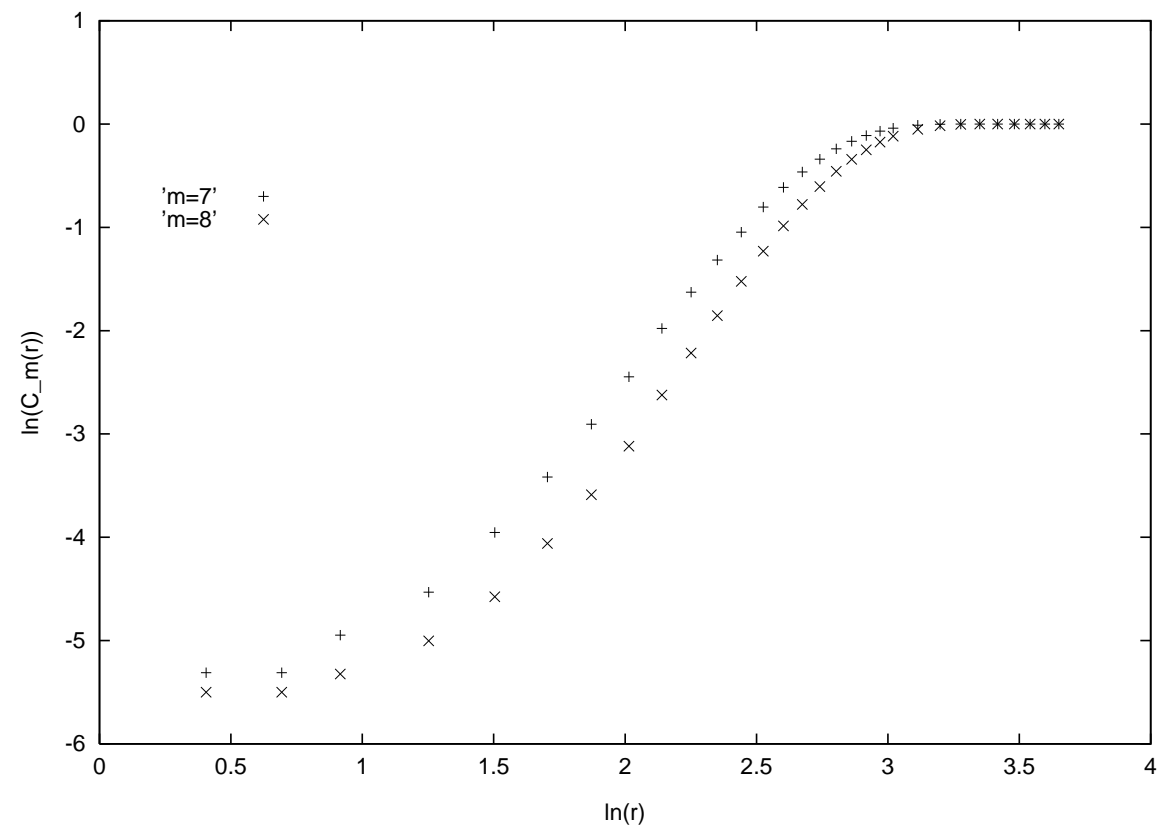

Figure 1: $\ln r-\ln C_{m}(r)$ figure of phage 5'UTR sequence when $\mathrm{m}=7,8$.

(Figure 1). We take the region from the third point to the 17th point (from left to right) as the scaling region

To study time series, Hurst 22 invented a new statistical method - the rescaled range analysis ( $R / S$ analysis), later on B. B. Mandelbrot 23] and J. Feder 24 transplanted $R / S$ analysis into fractal analysis. For any time series $x=\left\{x_{k}\right\}_{k=1}^{N}$ and any $2 \leq n \leq N$, one can define

$$
\begin{gathered}
<x>_{n}=\frac{1}{n} \sum_{i=1}^{n} x_{i} \\
X(i, n)=\sum_{u=1}^{i}\left[x_{u}-<x>_{n}\right] \\
R(n)=\max _{1 \leq i \leq n} X(i, n)-\min _{1 \leq i \leq n} X(i, n) \\
S(n)=\left[\frac{1}{n} \sum_{i=1}^{n}\left(x_{i}-<x>_{n}\right)^{2}\right]^{1 / 2} .
\end{gathered}
$$

Hurst found that

$$
R(n) / S(n) \sim\left(\frac{n}{2}\right)^{H} .
$$

$H$ is called Hurst exponent. 


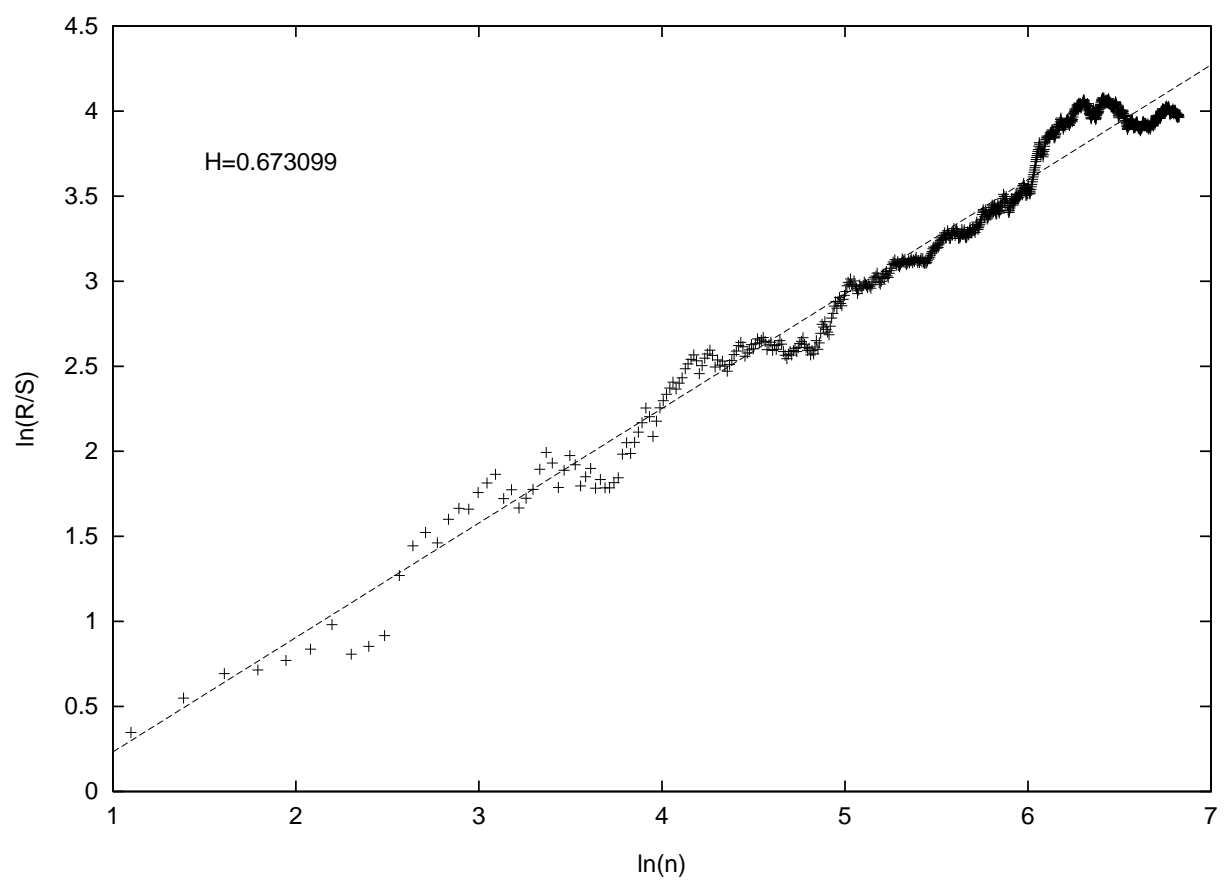

Figure 2: An example of $R / S$ analysis of DNA sequence

As $n$ changes from 2 to $N$, we obtain $N-1$ points in $\ln (n)$ v.s. $\ln (R(n) / S(n))$ plane. Then we can calculate Hurst exponent $H$ of DNA sequence $s$ using the least-square linear fit. As an example, we plot the graph of $R / S$ analysis of an exon segment $s$ of mouse' DNA sequence (bp 1730- bp 2650 of the record with Accession AF033620 in Genbank) in Figure 2.

The Hurst exponent is usually used as a measure of complexity. From Page 149 of Ref.[22], the trajectory of the record is a curve with a fractal dimension $D=2-H$. Hence a smaller $H$ means a more complex system. When applied to fractional Brownian motion, if $H>1 / 2$, the system is said to be persistent, which means that if for a given time period $t$, the motion is along one direction, then in the succeeding $t$ time, it's more likely that the motion will follow the same direction. While for system with $H<1 / 2$, the opposite holds, that is, antipersistent. But when $H=1 / 2$, the system is Brown motion, and is random.

\section{Data and results.}

More than 18 bacterial complete genomes are now available in public databases . There are four Archaebacteria: Archaeoglobus fulgidus (aful), Pyrococcus horikoshii (pyro), Methanococcus jannaschii (mjan), and Methanobacterium thermoautotrophicum (mthe); 
Table 1: Average of $D_{2}$ of genes of 18 bacteria.

\begin{tabular}{|c|l|l|}
\hline \hline Average of $D_{2}$ & species of Bacterium & Category \\
\hline 2.805 & Mycoplasma genitalium (mgen) & Gram-positive Eubacteria \\
2.827 & Methanococcus jannaschii (mjan) & Archaebacteria \\
2.872 & Rockettsia prowazekii (rpxx) & Proteobacteria \\
2.883 & Helicobacter pylori 26695 (hpyl) & Proteobacteria \\
2.908 & Helicobacter pylori J99 (hpyl99) & Proteobacteria \\
2.938 & Haemophilus influenzae (hinf) & Proteobacteria \\
2.940 & Mycoplasma pneumoniae (mpneu) & Gram-positive Eubacteria \\
2.950 & Mycobacterium tuberculosis (mtub) & Gram-positive Eubacteria \\
2.990 & Bacillus subtilis (bsub) & Gram-positive Eubacteria \\
\hline \hline 3.011 & Aquifex aeolicus (aquae) & hyperthermophilic bacterium \\
3.012 & Pyrococcus horikoshii (pyro) & Archaebacteria \\
3.013 & M. thermoautotrophicum & Archaebacteria (mthe) \\
3.016 & Archaeoglobus fulgidus (aful) & Archaebacteria \\
3.020 & Chlamydia trachomatis (ctra) & Chlamydia \\
3.024 & Chlamydia pneumoniae (cpneu) & Chlamydia \\
3.028 & Synechocystis PCC6803 (synecho) & Cyanobacteria \\
3.047 & Rhizobium sp. NGR234 (pNGR234) & Proteobacteria \\
3.060 & Escherichia coli (ecoli) & Proteobacteria \\
\hline \hline
\end{tabular}

four Gram-positive Eubacteria: Mycobacterium tuberculosis (mtub), Mycoplasma pneumoniae (mpneu), Mycoplasma genitalium (mgen), and Bacillus subtilis (bsub). The others are Gram-negative Eubacteria: one hyperthermophilic bacterium Aquifex aeolicus (aquae); six proteobacteria: Rhizobium sp. NGR234 (pNGR234), Escherichia coli (ecoli), Haemophilus influenzae (hinf), Helicobacter pylori J99 (hpyl99), Helicobacter pylori 26695 (hpyl) and Rockettsia prowazekii (rpxx); two chlamydia Chlamydia trachomatis (ctra) and Chlamydia pneumoniae (cpneu), and one cyanobacterium Synechocystis PCC6803 (synecho).

For a given bacterium, we calculate the correlation dimension and Hurst exponent of each CDS sequence (i.e. the coding sequence) in its complete genome first (the results is shown in Figure 3 and Figure 4), then calculate the average of these two quantities. We find that the average of Hurst exponents of 18 bacteria are almost equal (range being from 0.538 to 0.590 ). But the differences among the values of average of correlation dimensions of these bacteria are larger. One can see Table 1 (from top to bottom, the value of $D_{2}$ 
become larger).

\section{Discussion and conclusions}

Although the existence of the archaebacterial urkingdom has been accepted by many biologists, the classification of bacteria is still a matter of controversy [25]. The evolutionary relationship of the three primary kingdoms (i.e. archeabacteria, eubacteria and eukaryote) is another crucial problem that remains unresolved 25.

From Table 1, we can roughly divide bacteria into two class first, the average of $D_{2}$ of one class is less than 3.0, that of another class is greater than 3.0. We can see that the classification of bacteria using the average of $D_{2}$ is almost coincide with the original classification of bacteria. Archaebacteria gather with each other except mjan. Grampositive bacteria get together except mgen. Chlamydia also gather with each other. Proteobacteria is divided into two sub-category: rpxx, hpyl, hpyl99 and hinf belong to one sub-category; pNGR234 and ecoli belong another sub-category.

A surprising feature shown in Table 1 is that Aquifex aeolicus is linked closely with the Archaebacteria. We noticed that Aquifex, like most Archaebacteria, is hyperthermophilic. It has previously been shown that Aquifex has close relationship with Archaebacteria from the gene comparison of an enzyme needed for the synthesis of the amino acid trytophan [26]. Our result, from the comparison of the complete genome, shows that the case is even more worse. Such strong correlation on the level of complete genome between Aquifex and Archaebacteria is not easily accounted for by lateral transfer and other accidental events[26].

We calculate the average of correlation dimensions and Hurst exponents of genes in all 16 chromosome of Saccharomyces cerevisiae (yeast), they are 3.018 and 0.579 respectively. From Table 1, one can see that Archaebacteria and Chlamydia are linked more closely with yeast which belongs to eukaryote than other category of bacteria. There are several reports (such as Ref. [27]), in some RNA and protein species, archeabacteria are much more similar in sequences to eukaryotes than to eubacteria. Our present result supports this point of view.

We also randomly produce a sequence of length 3000 consisting of symbols from the alphabet $\{A, T, G, C\}$. The correlation dimension is 1.02883. From Table 1, Fig. 3, we can conclude that all CDS sequences are far from random sequences. Since the Hurst exponent of random sequence is 0.5 . From Fig. 4, we can see that correlation dimension is well than Hurst exponent when we compare real DNA sequence with a random sequence on the alphabet $\{A, T, G, C\}$.

In Ref. [14], we find the Hurst exponent is a good exponent to distinct different 
functional regions, but now we only consider the same kind of functional region (i.e. they are all genes), it is reasonable that the average of Hurst exponent do not change much. Fortunately, now we can use the average of correlation dimension to distinguish different species.

\section{ACKNOWLEDGMENTS}

The authors would like to express their thanks to Prof. Bai-lin Hao for reading the manuscript carefully, encouragement and many good suggestions.

\section{References}

[1] W. Li and K. Kaneko, Europhys. Lett. 17 (1992) 655.

[2] A. Grosberg, Y. Rabin, S. Havlin, and A. Neer, Europhys. Lett. 23 (1993) 373.

[3] (a) R. Voss, Phys. Rev. Lett. 68 (1992) 3805; (b) Fractals 2 (1994) 1.

[4] C.K. Peng, S. Buldyrev, A.L.Goldberg, S. Havlin, F. Sciortino, M. Simons, and H.E. Stanley, Nature 356 (1992) 168.

[5] H.E. Stanley, S.V. Buldyrev, A.L. Goldberg, Z.D. Goldberg, S. Havlin, R.N. Mantegna, S.M. Ossadnik, C.K. Peng, and M. Simons, Physica A 205 (1994) 214.

[6] H.Herzel, W. Ebeling, and A.O. Schmitt, Phys. Rev. E 50 (1994) 5061.

[7] P. Allegrini, M. Barbi, P. Grigolini, and B.J. West, Phys. Rev. E 52 (1995) 5281.

[8] S. V. Buldyrev, N.V. Dokholyan, A.L. Goldberger, S. Havlin, C.-K. Peng, H.E. Stanley and G.M. Visvanathan, Physica A 249 (1998) 430-438.

[9] Liaofu Luo, Weijiang Lee, Lijun Jia, Fengmin Ji and Lu Tsai, Phys. Rev. E 58(1) (1998) 861-871.

[10] D.B. Searls, Computer Appl. in Biosciences (CABIOS) 13 (1997) 333-344.

[11] L. F. Lou, Tsai Li, and Y. M. Zhou, J. Theor. Biol. 130 (1988) 351.

[12] L.F. Luo and L. Tsai, Chin. Phys. Lett. 5 (1988) 421-424.

[13] Zu-Guo Yu, Bai-lin Hao, Hui-min Xie and Guo-Yi Chen, Dimension of fractals related to language defined by tagged strings in complete genome. Chaos, Solitons and Fractals (Accepted for publication). 
[14] Zu-Guo Yu and Guo-Yi Chen, Rescaled range and transition matrix analysis of DNA sequences. Communication of Theor. Phys. (Accepted for publication).

[15] Ruqun Shen, Rensheng Chen, Lunjiang Lin, Jian Sun, Yi Xiao, and Jun Xu, Chinese Science Bulletin (in Chinese) 38 (1993) 1995-1997.

[16] C. M. Fraser et al., The minimal gene complement of Mycoplasma genitalium, Science, 270 (1995) 397.

[17] Bai-lin Hao, Hoong-Chien Lee, and Shu-yu Zhang, Fractals related to long DNA sequences and complete genomes, Chaos, Solitons and Fractals, to appear (1999).

[18] Bai-Lin Hao, Hui-Ming Xie, Zu-Guo Yu and Guo-Yi Chen, Avoided strings in bacterial complete genomes and a related combinatorial problem. Ann. of Combinatorics., to appear (1999).

[19] P. Grassberger and I. Procaccia, Physica D 9 (1983) 189.

[20] P. Grassberger and I. Procaccia, Phys. Rev. Lett. 50 (1983) 346.

[21] Hao Bai-lin, Elementary Symbolic Dynamics and Chaos in Dissipative Systems. World Scientific, Singapore, 1989.

[22] H.E. Hurst, Long-term storage capacity of reservoirs, Trans. Amer. Soc. Civ. Eng. 116 (1951) 770-808.

[23] B.B. Mandelbrot, The Fractal Geometry of Nature, W. H. Freeman, New York, 1982.

[24] J. Feder, Fractals, Plenum Press, New York, London, 1988.

[25] N. Iwabe et al, Evolutionary relationship of archeabacteria,eubacteria, and eukaryotes infer from phylogenetic trees of duplicated genes. Proc. Natl. Acad. Sci. USA 86 (1989) 9355-9359.

[26] E. Pennisi, Genome data shake the tree of life, Science 286 (1998) 672.

[27] K. Lechner, G. Heller \& A. Böck, Nucleic Acids Res. 16 (1988) 7817-7826. 

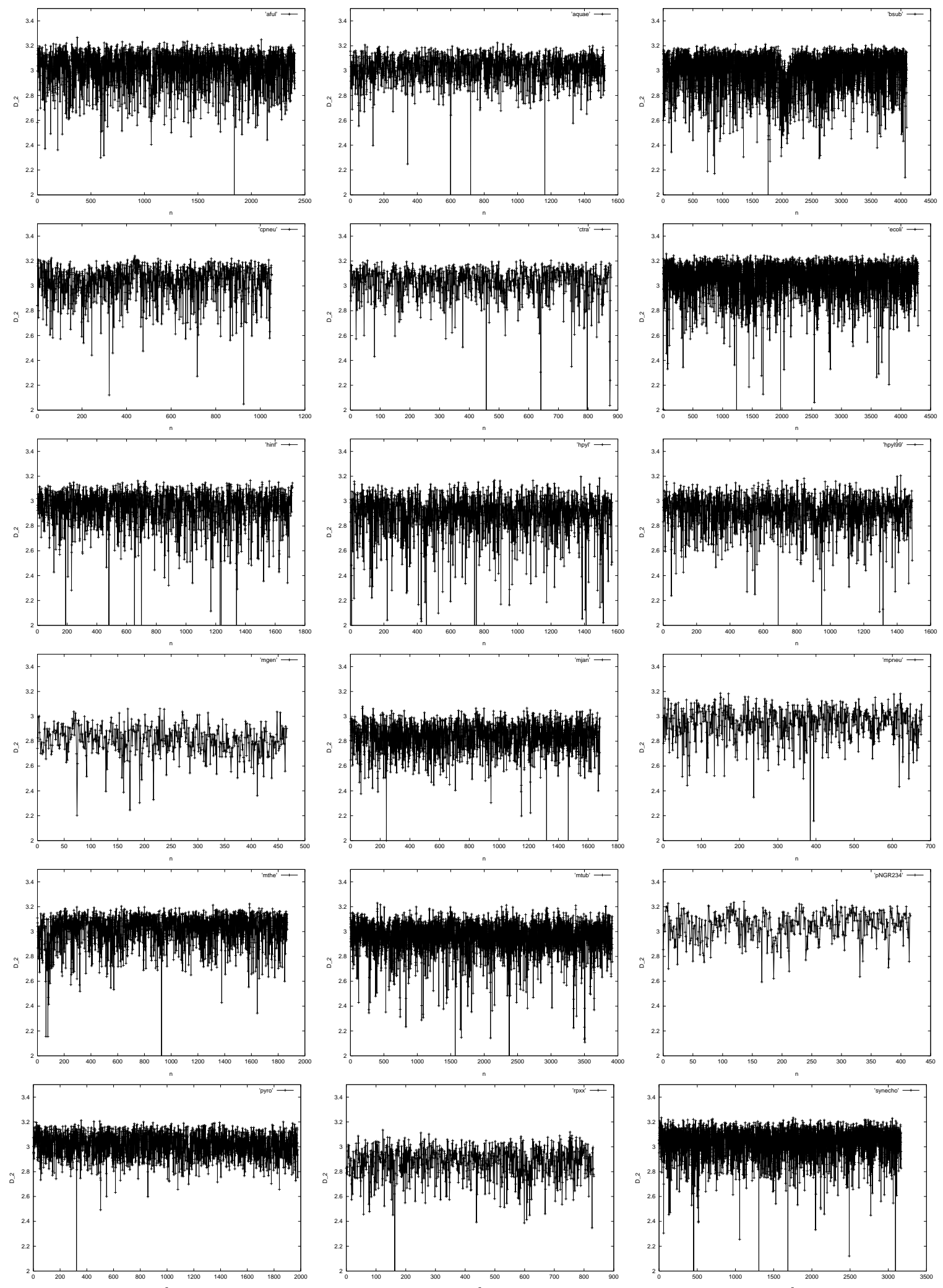

Figure 3: The correllation dimensions of CDS sequences in the complete genome of 18 bacteria. 

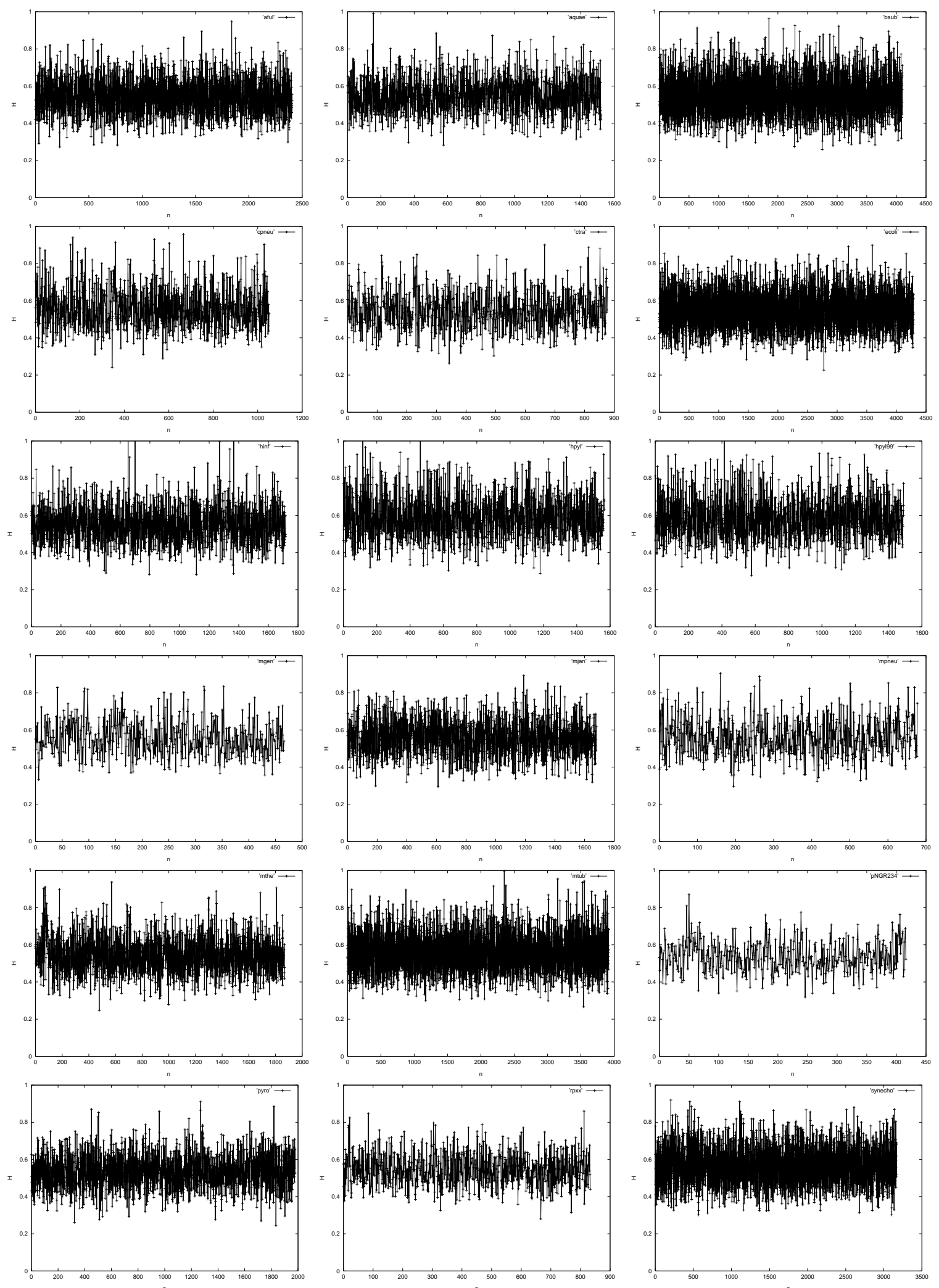

Figure 4: The Hurst exponents of CDS sequences in the complete genome of 18 bacteria. 